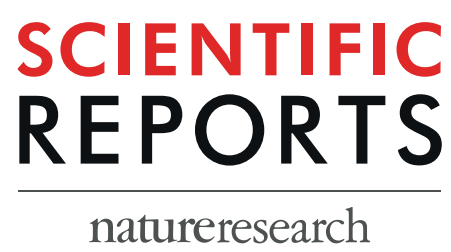

\title{
OPEN MicroRNA profiles in serum samples from Acute-On-Chronic Liver Failure patients and miR- 25-3p as a potential biomarker for survival prediction
}

\begin{abstract}
Júlia Cisilotto ${ }^{1}$, Alex Evangelista do Amaral ${ }^{1}$, Daiane Rosolen ${ }^{1}$, Michele Patrícia Rode ${ }^{1}$, Adny Henrique Silva ${ }^{2}$, Evelyn Winter ${ }^{3}$, Telma Erotides da Silva ${ }^{4}$, Josiane Fischer ${ }^{4}$, Camila Matiollo ${ }^{4}$, Elayne Cristina de Morais Rateke ${ }^{4}$, Janaína Luz Narciso-Schiavon ${ }^{4}$, Leonardo de Lucca Schiavon $\mathbb{1}^{4^{*}}$ \& Tânia Beatriz Creczynski-Pasa $\mathbb{( I D}^{1^{\star}}$
\end{abstract}

Acute-on-chronic liver failure (ACLF) is a condition characterized by acute decompensation of cirrhosis, associated with organ failure(s), and high short-term mortality. The microRNAs or miRNAs are small non-coding RNA molecules, stable in circulating samples such as biological fluids, and the difference in expression levels may indicate the presence, absence and/or stage of the disease. We analyzed here the miRNA profiling to identify potential diagnostic or prognostic biomarkers for ACLF. The major miRNAs discovered were validated in a cohort of patients with acute decompensation of cirrhosis grouped in no ACLF or ACLF according to EASL-CLIF definition. Relationship between serum miRNAs and variables associated with liver-damage and survival outcomes were verified to identify possible prognostic markers. Our results showed twenty altered miRNAs between no ACLF and ACLF patients, and twentyseven in patients who died in 30 days compared with who survived. In validation phase, miR-223-3p and miR-25-3p were significantly altered in ACLF patients and in those who died in 30 days. miR-223-3p and miR-25-3p expression were associated with the lowest survival in 30 days. The decrease in miR-223-3p and miR-25-3p expression was associated with the presence of ACLF and poor prognosis. Of these, miR25-3p was independently related to ACLF and 30-day mortality.

The natural history of cirrhosis is usually characterized by a long-standing compensated phase followed by a transition to the decompensated disease, identified by the occurrence of specific complications of cirrhosis, such as ascites, variceal bleeding, and hepatic encephalopathy ${ }^{1}$. Patients with both compensated or decompensated cirrhosis are at risk of progression to acute-on-chronic liver failure (ACLF), a condition characterized by an acute deterioration of the liver function and characterized by progression to extrahepatic organ failure and high short-term mortality ${ }^{2,3}$. Although a precise definition is still lacking, the European Association for the Study of the Liver-Chronic Liver Failure (EASL-CLIF) Consortium definition is one of the most validated criteria for ACLF in patients with cirrhosis and it is based on a modified version of SOFA score called CLIF-SOFA ${ }^{2,4}$. According to a recently published study ${ }^{5}$, mortality rates in ACLF patients are $25.5 \%$ and $40 \%$ in 28 and 90 days of admission, respectively. Therefore, searching for new biomarkers associated with the presence of ACLF and prognosis of patients with acute decompensation of cirrhosis may improve clinical decision and help to implement risk-adapted treatment strategies. In recent years, miRNAs have been studied as promising biomarkers for the diagnosis and prognostic in many clinical scenarios ${ }^{6-8}$, including liver diseases ${ }^{9}$. The miRNAs are a group of small non-coding RNAs, with approximately 22 nucleotides, which post-transcriptionally regulate gene expression

${ }^{1}$ Department of Pharmaceutical Sciences, Federal University of Santa Catarina, Florianopolis, 88040-900, SC, Brazil. ${ }^{2}$ Department of Biochemistry, Federal University of Santa Catarina, Florianopolis, 88040-900, SC, Brazil. ${ }^{3}$ Department of Agriculture, Biodiversity and Forestry, Federal University of Santa Catarina, Curitibanos, 89520-000, SC, Brazil. ${ }^{4}$ Department of Internal Medicine, Division of Gastroenterology, Federal University of Santa Catarina, Florianópolis, 88040-900, SC, Brazil.*email: leo-jf@uol.com.br; tania.pasa@ufsc.br 


\begin{tabular}{|c|c|c|c|}
\hline Variables & & Patients (\%) & Values Mean \pm SD \\
\hline Age, years & & $35(100.0)$ & $53.6 \pm 8.8$ \\
\hline \multirow[t]{2}{*}{ Gender } & Male & $26(74.3)$ & \\
\hline & Female & $9(25.7)$ & \\
\hline \multicolumn{4}{|l|}{ Etiology of cirrhosis } \\
\hline \multirow{2}{*}{ Hepatitis C } & Male & $10(28.6)$ & \\
\hline & Female & $4(11.4)$ & \\
\hline \multirow{2}{*}{ Hepatitis B } & Male & $2(5.7)$ & \\
\hline & Female & $1(2.9)$ & \\
\hline \multirow{2}{*}{ Alcohol } & Male & $21(60.0)$ & \\
\hline & Female & $1(2.9)$ & \\
\hline ACLF & & $20(57.1)$ & \\
\hline Bacterial infection (first $\mathbf{4 8 h}$ ) & & $16(45.7)$ & \\
\hline Ascites at admission & & $21(60.0)$ & \\
\hline \multicolumn{4}{|l|}{ Medications at admission } \\
\hline Propranolol & & $15(42.9)$ & \\
\hline Spironolactone & & $15(42.9)$ & \\
\hline Furosemide & & $12(34.3)$ & \\
\hline Lactulose & & $10(28.6)$ & \\
\hline Omeprazole & & $14(40.0)$ & \\
\hline Oral hypoglycemic* & & $4(11.4)$ & \\
\hline Insulin & & $3(8.6)$ & \\
\hline Death in 30 days & & $7(20.0)$ & \\
\hline \multicolumn{4}{|l|}{ Biochemical data } \\
\hline Total bilirubin (mg/dL) & & $35(100.0)$ & $4.9 \pm 7.9$ \\
\hline INR & & $33(94.3)$ & $1.5 \pm 0.3$ \\
\hline $\mathrm{CRP}(\mathrm{mg} / \mathrm{L})$ & & $28(80.0)$ & $35.0 \pm 45.8$ \\
\hline Creatinine $(\mathrm{mg} / \mathrm{dL})$ & & $26(74.3)$ & $1.5 \pm 0.8$ \\
\hline $\operatorname{Albumin}(\mathrm{g} / \mathrm{dL})$ & & $34(97.1)$ & $2.4 \pm 0.5$ \\
\hline Sodium $(\mathrm{mEq} / \mathrm{L})$ & & $33(94.3)$ & $134.4 \pm 4.9$ \\
\hline Total leukocyte $\left(\mathrm{mm}^{3}\right)$ & & $32(91.4)$ & $7163.8 \pm 5923.0$ \\
\hline
\end{tabular}

Table 1. Characteristics of the included patients in miRNA microarray analysis. ACLF. acute-on-chronic liver failure; SD. standard deviation; INR. international normalized ratio; CRP. C-reactive protein. *Metformin, glibenclamide and glimepiride.

usually by binding to the $3^{\prime}$ UTR of the mRNA ${ }^{10,11}$. The miRNAs have already been detected in different biological fluids such as plasma, serum, tears, breast milk, cerebrospinal fluid, saliva, urine, and others ${ }^{12,13}$. It has been shown that miRNAs are stable in serum/plasma and can be directly detected in these fluids, which improve their application as biomarkers. In addition, miRNAs are resistant to RNaseA digestion and other harsh conditions, which potentially support its usage in clinical test ${ }^{14}$.

Although there are data regarding different miRNAs in patients with hepatitis B virus (HBV)-related $\mathrm{ACLF}^{15,16}$, to our knowledge, no study investigated miRNAs in patients with acute decompensation of cirrhosis or ACLF. Thus, in the present study, we aimed to evaluate the miRNAs expression in serum samples from patients with acute decompensation of cirrhosis, identifying possible biomarkers of ACLF and prognosis.

\section{Results}

miRNA selection from the microarray panel. At the first phase, 35 serum samples were evaluated in miRNAs microarray analysis. The mean age of these patients was $53.6 \pm 8.8$ years, with male predominance (74.3\%). The most common etiologic factor of cirrhosis was alcohol consumption (62.9\%) followed by hepatitis C (40.0\%). Upon admission, ascites was observed in $60.0 \%$ of cases and bacterial infection in $45.7 \%$. The most frequent medications regularly used by the included subjects were propranolol (42.9\%), spironolactone (42.9\%), furosemide (34.3\%), lactulose (28.6\%) and omeprazole (40.0\%). Among the 35 patients, 20 fulfilled the criteria for ACLF and 7 died within 30 days (Table 1).

As showed in Table 2, miRNA expression from microarray analysis was evaluated according to the presence of ACLF and 30-day mortality. The expression of 20 miRNAs was decreased by at least 6-fold in ACLF patients compared to no ACLF (fold-change (FC) $\geq 6$ ). Among them, expression of the miRNAs let-7a-5p, let-7g-5p, miR-106b-5p, miR-107, miR-126-3p, miR-17-5p, miR-20a-5p, miR-25-3p and miR-451a was decreased by at least 10 -fold in ACLF patients $(F C \geq 10)$. When miRNA expression was evaluated according to survival, expression of 27 miRNAs was reduced in those who died within 30 days $(F C \geq 6)$. Expression of miRNAs let-7a-5p, let-7g-5p, miR-106b-5p, miR-126-3p, miR-15b-5p, miR-17-5p, miR-199a-3p, miR-20a-5p, miR-223-3p, miR-25-3p and miR-575 were decreased by at least 10 -fold in individuals who died in relation to survivors (FC $\geq 10$ ). 


\begin{tabular}{|c|c|c|c|c|c|c|c|}
\hline \multicolumn{4}{|c|}{ no ACLF vs ACLF patients } & \multicolumn{4}{|c|}{ Survival $v s$ Death in 30 days } \\
\hline miRNA & P-value & FC & Regulation & miRNA & P-value & FC & Regulation \\
\hline let-7a-5p & 0.004 & 10 & down & let-7a-5p & 0.009 & 13 & down \\
\hline let-7b-5p & 0.007 & 6 & down & let-7f-5p & 0.012 & 9 & down \\
\hline let- $7 g-5 p$ & 0.009 & 14 & down & let- $7 g-5 p$ & 0.008 & 18 & down \\
\hline miR-106b-5p & 0.007 & 10 & down & miR-106b-5p & 0.006 & 13 & down \\
\hline miR-107 & 0.001 & 11 & down & miR-126-3p & 0.004 & 14 & down \\
\hline miR-126-3p & 0.006 & 14 & down & miR-146a-5p & 0.012 & 9 & down \\
\hline miR-142-3p & 0.009 & 8 & down & miR-150-5p & 0.015 & 8 & down \\
\hline miR-150-5p & 0.005 & 7 & down & miR-15b-5p & 0.017 & 10 & down \\
\hline miR-15b-5p & 0.002 & 9 & down & miR-16-5p & 0.026 & 8 & down \\
\hline miR-16-5p & 0.006 & 8 & down & miR-17-5p & 0.011 & 12 & down \\
\hline miR-17-5p & 0.004 & 15 & down & miR-199a-3p & 0.009 & 10 & down \\
\hline miR-20a-5p & 0.012 & 18 & down & miR-19a-3p & 0.013 & 8 & down \\
\hline miR-223-3p & 0.002 & 7 & down & miR-19b-3p & 0.021 & 7 & down \\
\hline miR-25-3p & 0.005 & 16 & down & miR-20a-5p & 0.043 & 15 & down \\
\hline miR-27a-3p & 0.008 & 7 & down & miR-223-3p & 0.003 & 10 & down \\
\hline miR-328-5p & 0.003 & 6 & down & miR-23a-3p & 0.011 & 8 & down \\
\hline miR-451a & 0.004 & 10 & down & miR-24-3p & 0.092 & 9 & down \\
\hline miR-4530 & 0.002 & 7 & down & miR-25-3p & 0.026 & 11 & down \\
\hline miR-5703 & 0.001 & 7 & down & miR-328-5p & 0.017 & 6 & down \\
\hline \multirow[t]{8}{*}{ miR-630 } & 0.001 & 7 & down & miR-4515 & 0.018 & 8 & down \\
\hline & & & & miR-451a & 0.029 & 8 & down \\
\hline & & & & miR-4530 & 0.009 & 7 & down \\
\hline & & & & miR-4741 & 0.004 & 7 & down \\
\hline & & & & miR-575 & 0.010 & 10 & down \\
\hline & & & & miR-5787 & 0.019 & 7 & down \\
\hline & & & & miR-6090 & 0.019 & 7 & down \\
\hline & & & & miR-6752-5p & 0.008 & 6 & down \\
\hline
\end{tabular}

Table 2. miRNA expression from microarray analysis according to the presence of ACLF and 30-day mortality. It was considered only miRNAs with fold-change $(\mathrm{FC}) \geq 6$ and $\mathrm{P}$-value $<0.05$.

When data of survivors' patients and those who died within 30 days were compared using a FC less than 6 it was possible to detect the following upregulated miRNAs: miR-1180-3p $(\mathrm{FC}=3 ; \mathrm{P}=0.02)$; miR-1228-5p $(\mathrm{FC}=3 ; \mathrm{P}=0.02) ; \mathrm{miR}-670-5 \mathrm{p}(\mathrm{FC}=3 ; \mathrm{P}=0.04)$; miR-6847-3p $(\mathrm{FC}=3 ; \mathrm{P}=0.02) ;$ miR-7844-5p $(\mathrm{FC}=3$; $\mathrm{P}=0.04)$. Figure 1 shows the expression intensity of deregulated miRNAs $(\mathrm{FC} \geq 6$ and $\mathrm{P}<0.05)$ in samples from patients with no ACLF, ACLF, and those who died. ACLF patients exhibited a reduction in expression intensity mainly in the miRNAs let-7a-5p, let-7g-5p, miR-106b-5p, miR-107, miR-126-3p, miR-142-3p, miR-150-5p, miR-17-5p, miR-20a-5p, miR-25-3p and miR-27a-3p. It was also checked whether the most frequent medications regularly used by the patients (propranolol, spironolactone, furosemide, lactulose, norfloxacin, omeprazole, oral hypoglycemic and insulin) would alter miRNA expression. As can be seen in Supplementary Fig. S1, no medication-related changes in miRNAs expression $(\mathrm{P}<0.05$ and $\mathrm{FC}>2)$ were observed.

Based on the magnitude of expression difference between patients with and without ACLF in the microarray analysis and in literature reports ${ }^{17-23}$ miRNAs miR-106b-5p, miR-126-3p, miR-20a-5p, miR-223-3p, and miR$25-3 p$ were selected to be further validated by RT-qPCR. Some miRNAs that had high FC values such as let-7a-5p and let-7g-5p were not detected in most microarray samples and were therefore not included in the validation step.

Characteristics of included patients and associations between miRNA expression and variables of interest. One hundred and thirty-nine subjects with acute decompensation of cirrhosis were included in the validation step for RT-qPCR. Table 3 shows the main characteristics of patients. The mean age was $55.3 \pm 11.2$ years and a male predominance was observed (70.5\%). The most common etiology of cirrhosis was alcohol abuse (50.4\%) followed by hepatitis C (37.4\%), and hepatitis B (7.2\%). The main medications regularly used by patients prior to the blood sampling were propranolol (36.7\%), spironolactone (31.7\%), furosemide (21.6\%), lactulose (20.9\%), norfloxacin (11.5\%) and omeprazole (35.5\%). The mean MELD score was $17.5 \pm 6.9$ and 35 patients (25.1\%) fulfilled ACLF criteria.

When miR-106b-5p, miR-126-3p, miR-20a-5p, miR-223-3p and miR-25-3p were evaluated according to the study variables, the expression of miR-223-3p and miR-25-5p was significantly decreased in patients with hepatic encephalopathy at admission $(\mathrm{P}=0.005$ and 0.034 , respectively) and in ACLF individuals $(\mathrm{P}=0.026$ and 0.009, respectively; Supplementary Table S1 and Fig. 2). Moreover, when evaluating only ACLF patients $(\mathrm{n}=35)$, no significant differences were observed in the expressions of miR-25-3p (median:10.3 vs 13.4, respectively; $\mathrm{P}=0.250)$ and miR-223-3p (median: 28.1 vs 64.8 , respectively; $\mathrm{P}=0.117)$ among those with $(\mathrm{n}=26$, $74.3 \%)$ and without hepatic encephalopathy. Similarly, when only no ACLF patients $(\mathrm{n}=104)$ were considered, 


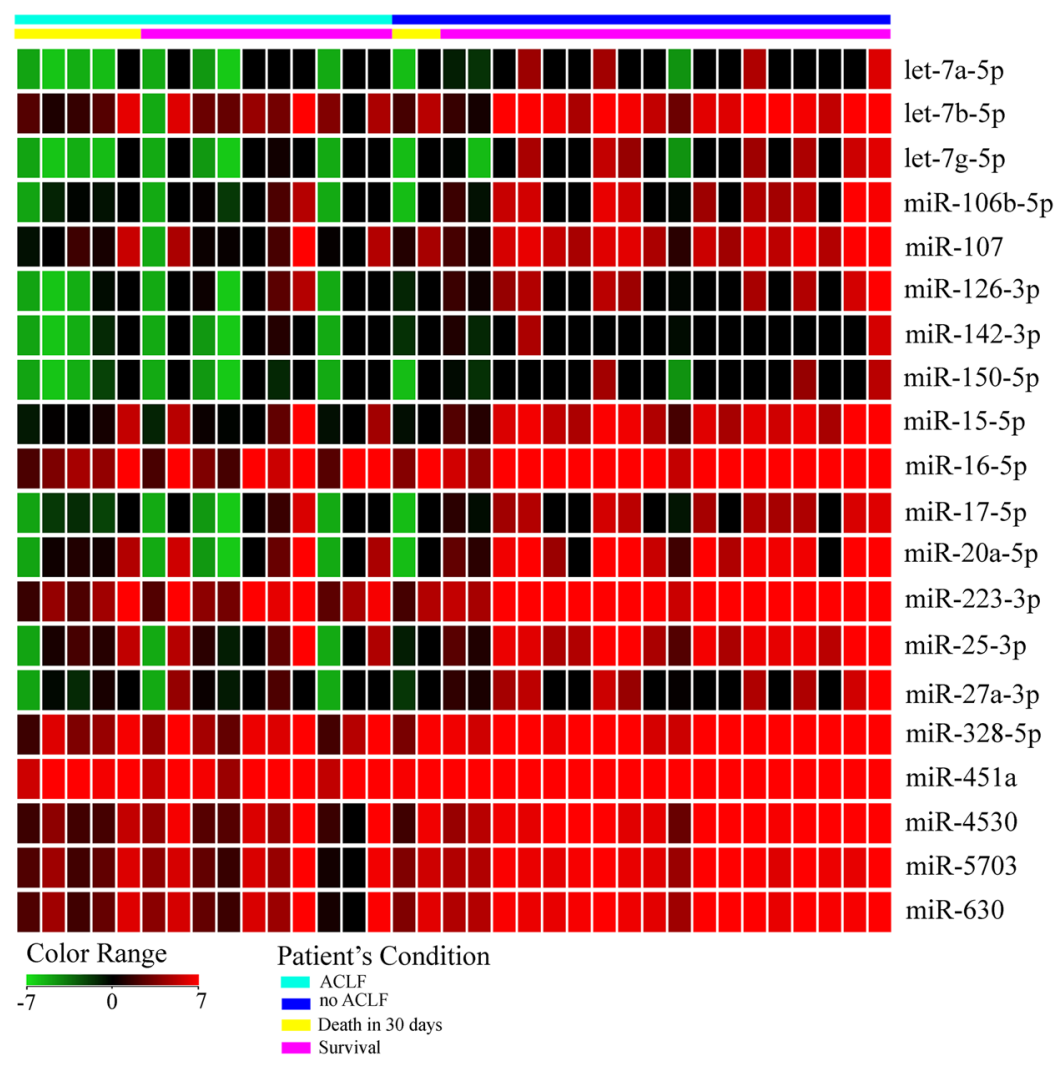

Figure 1. Heat map of differentially expressed miRNAs according to the presence of ACLF and survival. Heat map colors correspond to the level of miRNA expression as indicated in the color range.

no differences were observed for miR-25-3p (median: 42.7 vs 56.3, respectively; $\mathrm{P}=0.352$ ) and miR-223-3p (median: 56.7 vs 90.6, respectively; $\mathrm{P}=0.092)$ among those with $(\mathrm{n}=37,35.6 \%)$ and without hepatic encephalopathy. Regarding correlation analysis, miR-106b-5p negatively correlated with total leukocyte count $(r=-0.212$, $\mathrm{P}=0.012)$ and miR-20a-5p negatively correlated with serum creatinine $(\mathrm{r}=-0.212, \mathrm{P}=0.012) . \mathrm{miR}-223-3 \mathrm{p}$ expression was negatively correlated with age $(\mathrm{r}=-0.193, \mathrm{P}=0.025)$, creatinine levels $(\mathrm{r}=-0.302, \mathrm{P}<0.001)$, and CLIF-SOFA $(\mathrm{r}=-0.186, \mathrm{P}=0.034)$. Likewise, miR-25-3p expression was negatively correlated with serum creatinine $(\mathrm{r}=-0.341, \mathrm{P}<0.001)$, CLIF-SOFA $(\mathrm{r}=-0.196, \mathrm{P}=0.026)$, and MELD score $(\mathrm{r}=-0.185, \mathrm{P}=0.029$; Supplementary Table S2).

Factors associated with ACLF. As shown in Table 3, ACLF patients showed a higher prevalence of hepatic encephalopathy $(74.3 \%$ vs. $35.6 \%, \mathrm{P}<0.001)$ and ascites $(82.9 \%$ vs. $38.5 \%, \mathrm{P}<0.001)$, and lower prevalence of gastrointestinal bleeding ( $14.3 \%$ vs. $41.3 \%, \mathrm{P}=0.004)$. Regarding biochemical parameters, ACLF patients exhibited higher median creatinine $(\mathrm{P}<0.001)$, total bilirubin $(\mathrm{P}=0.029)$, INR $(\mathrm{P}=0.034)$, CRP $(\mathrm{P}=0.029)$, and lower mean sodium levels $(\mathrm{P}<0.001)$. ACLF was also related to lower mean arterial pressure $(\mathrm{P}=0.020)$ and higher MELD score $(\mathrm{P}<0.001)$. As described before, subjects with ACLF at admission exhibited decreased expression of miR-223-3p and miR-25-5p (Table S1).

A logistic regression analysis was performed to investigate factors independently associated with ACLF including the following covariates with $\mathrm{P}<0.05$ in the bivariate analysis: sodium levels, hepatitis $\mathrm{C}$, Child-Pugh $\mathrm{C}$, miR-223-3p and miR-25-3p expression. In this analysis, ACLF at admission was independently associated with lower sodium levels $(\mathrm{OR}=0.82$, 95\% CI: 0.74-0.91, $\mathrm{P}<0.001)$, Child-Pugh C (OR=3.48, 95\% CI: 1.34-9.01, $\mathrm{P}=0.010)$, hepatitis $\mathrm{C}(\mathrm{OR}=3.69,95 \% \mathrm{CI}: 1.35-10.04, \mathrm{P}=0.011)$ and miR-25-3p expression $(\mathrm{OR}=0.99,95 \%$ CI: 0.98-0.99, $\mathrm{P}=0.040)$.

miRNAs as prognostic markers. During the first 30 days of follow-up, 31 patients died (22\%). Survival analysis was performed to identify the association between studied miRNAs and 30-day mortality. Expressions of miR-223-3p (HR =0.99, 95\% CI: 0.98-0.99, $\mathrm{P}=0.030)$ and $\mathrm{miR}-25-3 \mathrm{p}(\mathrm{HR}=0.99,95 \% \mathrm{CI}: 0.98-0.99, \mathrm{P}=0.013)$ were associated with 30-day mortality in the univariate Cox regression analysis. As expected, Child-Pugh C $(\mathrm{HR}=3.99,95 \% \mathrm{CI}: 1.83-8.72, \mathrm{P}=0.001)$ and $\mathrm{ACLF}(\mathrm{HR}=4.78,95 \% \mathrm{CI}: 2.35-9.73, \mathrm{P}<0.001)$ were also related to shorter survival. Expressions of miR-106b-5p, miR-126-3p and miR-20a-5p were not associated with prognosis (Table 4). A multiple Cox regression analysis was conducted including the expression of miR-223-3p and miR-25-3p, along with Child-Pugh C and ACLF. As shown in Table 4, decreased miR-25-3p expression status $(\mathrm{HR}=0.99,95 \% \mathrm{CI}: 0.98-0.99, \mathrm{P}=0.044)$, Child-Pugh C $(\mathrm{HR}=2.92,95 \% \mathrm{CI}: 1.28-6.66, \mathrm{P}=0.011)$, and ACLF $(\mathrm{HR}=2.69,95 \% \mathrm{CI}: 1.25-5.80, \mathrm{P}=0.012)$ were independently predictors of 30 -day mortality. 


\begin{tabular}{|c|c|c|c|c|}
\hline & All $(n=139)$ & no $\operatorname{ACLF}(n=104)$ & $\operatorname{ACLF}(n=35)$ & P-value \\
\hline Age (years), mean \pm SD & $55.3 \pm 11.2$ & $55.4 \pm 11.8$ & $54.8 \pm 9.59$ & 0.783 \\
\hline Male Gender, $\mathbf{n}(\%)$ & $98(70.5)$ & $73(70.2)$ & $25(71.4)$ & 0.890 \\
\hline \multicolumn{5}{|l|}{\begin{tabular}{|l} 
Etiology of cirrhosis, $n(\%)$ \\
\end{tabular}} \\
\hline Hepatitis C & $52(37.4)$ & $34(32.7)$ & $18(51.4)$ & $0.048^{*}$ \\
\hline Hepatitis B & $10(7.2)$ & $7(6.7)$ & $3(8.6)$ & 0.715 \\
\hline Alcohol & $70(50.4)$ & $56(53.8)$ & $14(40.0)$ & 0.156 \\
\hline \multicolumn{5}{|l|}{\begin{tabular}{|l|} 
Complication at admission, $\mathbf{n}(\%)$ \\
\end{tabular}} \\
\hline Ascites & 69 (49.6) & 40 (38.5) & $29(82.9)$ & $<0.001 * * *$ \\
\hline Hepatic encephalopathy & $63(45.3)$ & $37(35.6)$ & $26(74.3)$ & $<0.001 * * *$ \\
\hline Gastrointestinal bleeding & $48(34.5)$ & $43(41.3)$ & $5(14.3)$ & $0.004 * *$ \\
\hline Bacterial infection & 69 (49.6) & $48(46.2)$ & $21(60)$ & 0.156 \\
\hline \multicolumn{5}{|l|}{ Medications at admission, $\mathbf{n}(\%)$} \\
\hline Propranolol & $51(36.7)$ & $42(40.4)$ & $9(25.7)$ & 0.119 \\
\hline Spironolactone & $44(31.7)$ & $30(28.8)$ & $14(40)$ & 0.220 \\
\hline Furosemide & $30(21.6)$ & $17(16.3)$ & $13(37.1)$ & $0.010^{*}$ \\
\hline Lactulose & $29(20.9)$ & $18(17.3)$ & $11(31.4)$ & 0.075 \\
\hline Norfloxacin & $16(11.5)$ & $8(7.7)$ & $8(22.9)$ & $0.015^{*}$ \\
\hline Omeprazole & $49(35.5)$ & $39(37.9)$ & $10(28.6)$ & 0.321 \\
\hline Oral hypoglycemic*• & $19(13.8)$ & $17(16.5)$ & $2(5.7)$ & 0.109 \\
\hline Insulin & $15(11.0)$ & $10(9.9)$ & $5(14.3)$ & 0.475 \\
\hline \multicolumn{5}{|l|}{\begin{tabular}{|l|} 
Child-Pugh Classification, n (\%) \\
\end{tabular}} \\
\hline Child-Pugh A & $11(10.6)$ & $11(8.0)$ & $0(0.0)$ & 0.065 \\
\hline Child-Pugh B & $71(51.4)$ & $60(57.7)$ & $11(32.4)$ & $0.017^{*}$ \\
\hline Child-Pugh C & $56(40.6)$ & $33(31.7)$ & $23(67.6)$ & $<0.001^{* * *}$ \\
\hline MELD score, mean \pm SD & $17.5 \pm 6.9$ & $14.8 \pm 4.4$ & $25.4 \pm 7.2$ & $<0.001^{* * *}$ \\
\hline \multicolumn{5}{|l|}{\begin{tabular}{|l|} 
Biochemical data \\
\end{tabular}} \\
\hline Creatinine $(\mathrm{mg} / \mathrm{dL})$, median & 1.1 & 1.0 & 2.4 & $<0.001$ \\
\hline Total leukocyte $\left(\mathrm{mm}^{3}\right)$, median & 6700.0 & 6540.0 & 8450.0 & 0.072 \\
\hline Total bilirubin $(\mathrm{mg} / \mathrm{dL})$, median & 2.1 & 2.0 & 3.5 & $0.029^{*}$ \\
\hline Albumin $(\mathrm{g} / \mathrm{dL})$, mean $\pm \mathrm{SD}$ & $2.4 \pm 0.6$ & $2.4 \pm 0.6$ & $2.4 \pm 0.6$ & 0.893 \\
\hline INR, median & 1.5 & 1.4 & 1.6 & $0.034^{*}$ \\
\hline Sodium $(\mathrm{mEq} / \mathrm{L})$, mean $\pm \mathrm{SD}$ & $134.9 \pm 5.5$ & $136.2 \pm 5.0$ & $131.5 \pm 5.5$ & $<0.001$ *** \\
\hline CRP (mg/L), median & 19.6 & 18.0 & 26.4 & $0.029^{*}$ \\
\hline MAP $(\mathrm{mmHg})$, mean \pm SD & $83.4 \pm 16.9$ & $85.4 \pm 15.5$ & $77.5 \pm 20.0$ & $0.020^{*}$ \\
\hline
\end{tabular}

Table 3. Characteristics of included patients and factors associated with ACLF at enrollment. ACLF. acuteon-chronic liver failure; SD. standard deviation; INR. international normalized ratio; CRP. C-reactive protein; MELD. model for end-stage liver disease; MAP. Mean Arterial Pressure. Of the 52 patients with hepatitis $\mathrm{C}$ as etiology of cirrhosis 39 (75\%) were male and 13 (25\%) female; Of the 10 patients with hepatitis B as etiology of cirrhosis 8 (80\%) were male and $2(20 \%)$ female; Of the 70 patients with alcohol use as etiology of cirrhosis 65 $(92.9 \%)$ were male and $5(7.1 \%)$ female. ${ }^{\bullet}$ Metformin, glibenclamide and glimepiride. $* \mathrm{P}<0.05$; $* * \mathrm{P}<0.01$ and $* * * \mathrm{P}<0.001$.

Figure 3 shows the Kaplan-Meier curves for mortality during the follow-up period, according to miR-25-3p expression categorized in 50 . The Kaplan-Meier survival probability at 30 days was $87 \%$ for patients with miR25-3p expression $\geq 50$ and $70 \%$ for those with miR-25-3p expression $<50$ (red dotted line) $(\mathrm{P}=0.012)$.

Bioinformatic analysis of the miR-25-3p. The results showed 1873 targets predicted for the miR-25-3p. Among the target genes, some are related with inflammation, such as, intercellular adhesion molecule 1 (ICAM-1 or CADM1); C-X-C motif chemokine 5 (CXCL5); TNF receptor-associated factor 3 (TRAF3); interleukin 8 (IL8); interleukin 6 signal transducer (IL6ST); interleukin 17 receptor D (IL17RD) and interleukin 15 (IL15). The Supplementary Fig. S2 shows some significant pathways $(\mathrm{P}<0.05)$ within the list of miR-25-3p related genes. Among 1873 genes, 32.3\% are associated with the vascular endothelial growth factor (VEGF) and the VEGF receptor (VEGFR) signaling network; 32.2\% and 32.3\% with interleukin 5 (IL5) and 3 (IL3) signaling events, respectively; $32.3 \%$ with interferon gamma (INF- $\gamma$ ); $8.2 \%$ with tumor necrosis factor (TNF) receptors and $33.8 \%$ with TNF-related apoptosis-inducing ligand (TRAIL) signaling pathway.

\section{Discussion}

Over the last decade, significant advances were achieved in definition and understanding of the pathogenesis of ACLF. However, data about the role of miRNAs in this context is still lacking. In this study, we sought to identify miRNAs related to the presence of ACLF and prognosis in patients hospitalized for acute decompensation 
$\mathrm{a}$

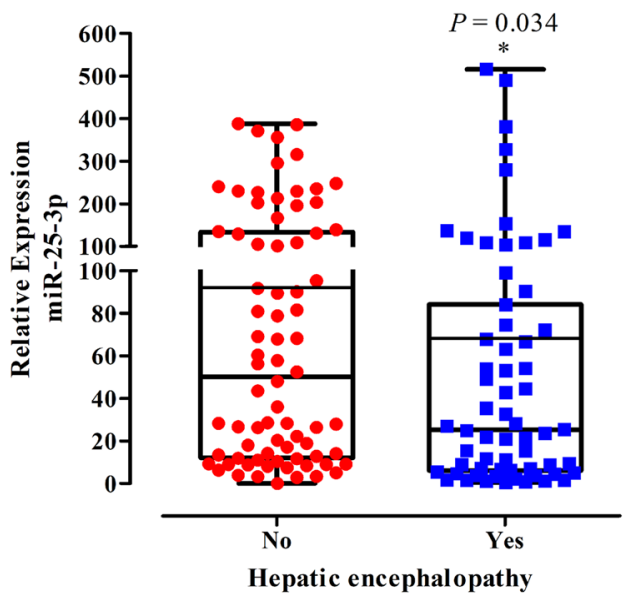

$\mathrm{C}$

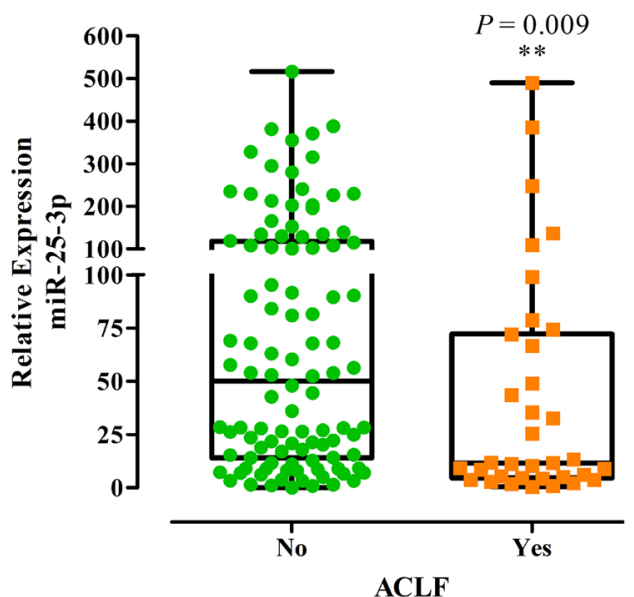

b

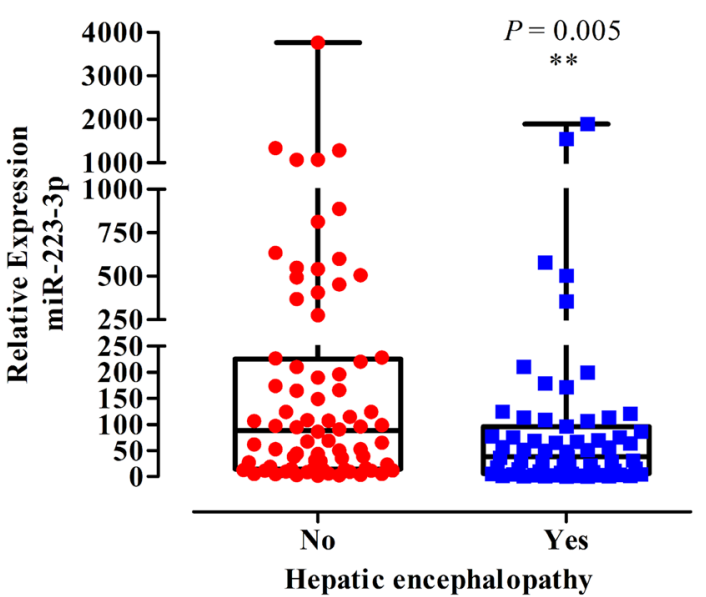

d

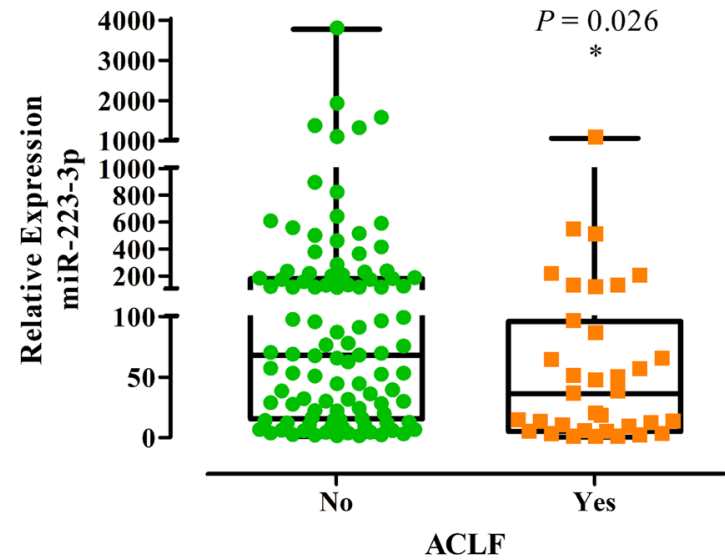

Figure 2. Relative expression of miR-25-3p and miR-223-3p in patients with hepatic encephalopathy (a,b) and $\mathrm{ACLF}$ patients $(\mathbf{c}, \mathbf{d})$. In the graphs, the horizontal lines of Box plot represent the median per group, minimum and maximum values. Statistical differences among the groups were calculated using Mann-Whitney U tests. Asterisks indicate whether there were significant differences among the groups. $* \mathrm{P}<0.05 ; * * \mathrm{P}<0.01$.

\begin{tabular}{|l|l|l|l|l|l|l|}
\hline \multirow{2}{*}{ Variables } & \multicolumn{4}{|l|}{ Univariate analysis } & \multicolumn{2}{l|}{ Multivariate analysis } \\
\cline { 2 - 7 } & HR & $\mathbf{9 5 \%}$ CI & P-value & HR & 95\% CI & P-value \\
\hline miR-106b-5p & 0.98 & $0.95-1.00$ & 0.051 & - & - & - \\
\hline miR-126-3p & 0.98 & $0.95-1.02$ & 0.279 & - & - & - \\
\hline miR-20a-5p & 0.99 & $0.99-1.00$ & 0.052 & - & - & - \\
\hline miR-223-3p & 0.99 & $0.98-0.99$ & 0.030 & - & - & - \\
\hline miR-25-3p & 0.99 & $0.98-0.99$ & 0.013 & 0.99 & $0.98-0.99$ & 0.044 \\
\hline Child-Pugh C & 3.99 & $1.83-8.72$ & 0.001 & 2.92 & $1.28-6.66$ & 0.011 \\
\hline ACLF & 4.78 & $2.35-9.73$ & $<0.001$ & 2.69 & $1.25-5.80$ & 0.012 \\
\hline
\end{tabular}

Table 4. Univariate and Multivariate Cox regression analysis of variables associated with 30-day survival among hospitalized patients with acute decompensation of cirrhosis. CI. confidence interval; HR. hazard ratio.

of cirrhosis. The first phase of the study aimed at evaluating the miRNAs panel differently expressed in ACLF patients by using a microarray-based approach, which allows the simultaneous evaluation of the countless miRNAs expression. In this analysis, it was possible to identify 20 altered miRNAs in ACLF patients and 27 miRNAs related to 30-day mortality. Among these, 5 miRNAs (miR-106b-5p; miR-126-3p; miR-20a-5p; miR-223-3p; miR25-3p) were selected for RT-qPCR validation. These miRNAs were chosen for validation based on (i) the results obtained in microarray analysis (P-value and fold-change); (ii) the number of samples in which a specific miRNA 


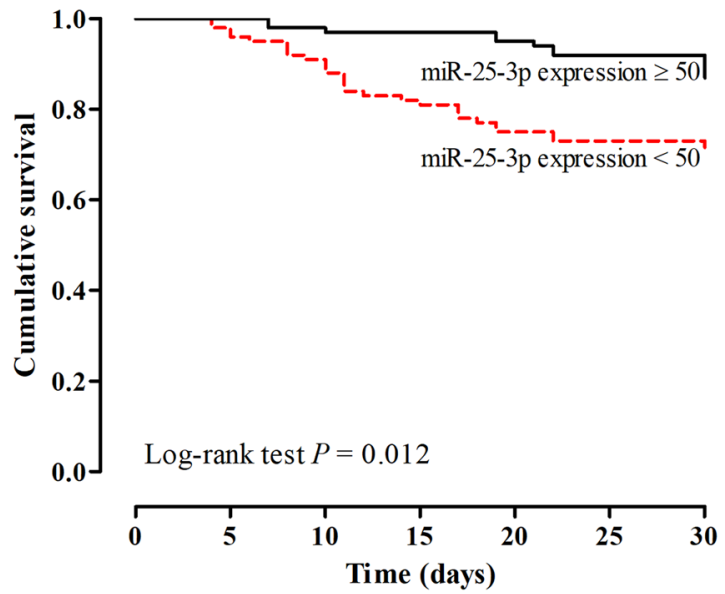

Figure 3. Cumulative 30-day survival of patients with cirrhosis according to miR-25-3p expression categorized in 50 . The Kaplan-Meier survival probability at 30 days was $87 \%$ for patients with miR-25-3p expression $\geq 50$ and $70 \%$ for those with miR-25-3p expression $<50$ (red dotted line) $(\mathrm{P}=0.012)$.

was detected; (iii) and previously published data regarding the miRNAs profile in decompensated cirrhosis ${ }^{17,18}$, inflammation ${ }^{19,20}$, and sepsis ${ }^{21-23}$.

When miRNAs serum expressions assessed by RT-qPCR were evaluated according to study variables, no relationship was observed between miR-126-3p and any of the analyzed parameters. There are no previous data regarding circulating miR-126-3p and liver cirrhosis. This miRNA was previously shown to be downregulated in non-cirrhotic patients with sepsis and was negatively correlated with inflammation biomarkers, blood urea nitrogen levels, and total bilirubin ${ }^{23}$. It is possible that microarray results regarding miR-126-3p reflect the inflammatory component of ACLF, even though we were not able to demonstrate any relationship between this miRNA and parameters such as leukocyte count and CRP. In the present study, we also observed no significant correlations between miR-106b-5p and most of the studied variables, except the leukocyte count. Contrary to our results, Jin et al. ${ }^{18}$ showed that miR-106b-5p was downregulated in plasma from patients with HBV-related cirrhosis classified as Child-Pugh B/C $(n=47)$ compared to Child-Pugh A patients $(n=53)$. This discrepancy may be, at least in part, related to the impact of the etiological factor of liver disease in circulating miRNAs. The Chinese study only included HBV-infected patients, whereas, in our cohort, this etiological factor was present in the minority of the patients. Circulating miR-20a-5p was also not related to most of the study variables, but a negative correlation was observed between this miRNA and creatinine levels. miR-20a-5p was also not previously studied in patients with liver cirrhosis. However, it was shown to be downregulated in chronic kidney disease, justifying the results found here ${ }^{24}$

According to the results of the miRNAs validated by RT-qPCR, only the miR-223-3p and miR-25-3p were downregulated in ACLF patients. The reason for these results may be due to the significant increase of the sample size in the validation step and by the higher sensitivity of RT-qPCR assay when compared to the microarray technique. The miR-25-3p was independently related to ACLF in the logistic regression analysis. Furthermore, miR223-3p and miR-25-3p were negatively correlated with CLIF-SOFA score. Previous studies have shown decreased expression of circulating miR-223 in patients with hepatocellular carcinoma ${ }^{25,26}$, liver cirrhosis ${ }^{26,27}$ and chronic hepatitis $\mathrm{C}^{26}$. Moreover, expression of serum miR-25 has been deregulated in patients with hepatocellular carcinoma ${ }^{28}$. As pointed by Wasmuth et al. ${ }^{29}$, patients with severe sepsis and ACLF show a similar degree of cellular immune depression. Interestingly, low expression of miR-25-3p and miR-223-3p has already been shown in septic patients and were also related to poor prognosis ${ }^{21,22}$. Wang et al. ${ }^{21}$ evaluated the expression of miRNAs in 214 serum samples from sepsis patients (117 survivors and 97 non-survivors based on 28-day mortality). Six miRNAs had altered expression in patients who died, including the miR-223-3p $(\mathrm{P}<0.001)$. The sensitivity and specificity of miR223-3p to distinguish surviving patients from those who died at 28 days was similar to the SOFA score, with areas under the ROC curve (AUC) of 0.748 and 0.782 , respectively. Additionally, Yao et al. ${ }^{22}$. showed that low expression of miR-25-3p was related to the severity of sepsis and 28-day mortality. Furthermore, the decreased miR-25-3p level was negatively correlated with the SOFA score, CRP, and procalcitonin level. In another study, the regulatory mechanism of miR-25-3p in sepsis-induced cardiomyocyte apoptosis was investigated. The researchers noted that the miR-25-3p expression was reduced in the serum of septic rats and in LPS-induced cardiomyocytes, while IL6 and TNF- $\alpha$ were increased. Furthermore, the miR-25-3p could inhibit LPS-induced cardiomyocyte apoptosis by regulating PTEN as well as reduce injury triggered by sepsis through inhibiting the inflammatory reaction ${ }^{30}$. The miR233 may influence macrophage polarization, differentiation of immune cells and inflammasome activation ${ }^{31}$. It was suggested that increased expression of neutrophilic miR-223 in mice may protect alcohol-induced liver injury and on the other hand downregulation of neutrophilic miR-223 in alcoholics may increase alcohol-mediated activation of neutrophils and liver injury in these subjects. In addition, the decrease of miR-233 expression increased IL-6 and $\mathrm{p} 47^{\text {phox }}$ expression in peripheral blood neutrophils from alcoholics ${ }^{32}$. It has been shown that miR-233 is a regulator of the progression of nonalcoholic steatohepatitis by targeting several inflammatory genes such as C-X-C motif chemokine 10 (CXCL10) and transcriptional co-activator with PDZ-binding motif (Taz) in hepatocytes ${ }^{33}$. The 
deregulation of miR-233, seen in our work, may be part of the pathogenesis of ACLF by macrophage polarization and alteration of cytokines involved in the inflammatory process.

In this study, miR-223-3p and miR-25-3p expression was negatively correlated with creatinine values and was also significantly decreased in patients with hepatic encephalopathy at admission. The miR-223-3p expression appears to be affected by renal diseases as its expression was shown to be reduced in both acute and chronic kidney diseases ${ }^{34,35}$. miR-25-3p was also shown to be downregulated in diabetic nephropathy, suggesting an influence of renal disease on its expression ${ }^{36}$. Although there are no previous studies examining the relationship between miR-223-3p, miR-25-3p, and hepatic encephalopathy, the association observed here indicate that expression of these miRNAs may be affected by the severity of the liver disease. However, kidney and cerebral dysfunction are both included in the ACLF definition. Therefore, as these miRNAs were selected from the microarray panel as ACLF markers, possibly the association with creatinine levels and hepatic encephalopathy merely reflects the severity of ACLF, as discussed above.

Expressions of miR-223-3p and miR-25-3p were associated with 30-day mortality in the univariate Cox regression analysis. However, only miR-25-3p expression remained an independent prognostic factor after adjusting for Child-Pugh classification and ACLF. In target prediction analysis it was observed that miR-25-3p might control the expression of genes related to inflammatory responses. The miR-25-3p possibily controls the translation of ICAM-1, IL6ST, IL8, TRAF3, IL15, among others. In addition, it has been observed that the predicted genes of miR-25-3p are involved with VEGF signaling pathways, TNF receptors, mTOR and TRAIL pathways. Cirrhosis-associated immune dysfunction (CAID) is an important feature of advanced liver disease and it is associated with progression to ACLF and high mortality ${ }^{37}$. The decrease in miR-25-3p expression in advanced cirrhosis may be responsible for an imbalance in the translation of some inflammation-related cytokines, contributing for CAID. On the other hand, a reduced miR-25-3p expression may result from systemic inflammation and, in this case, this miRNA would serve as a biomarker of immune dysfunction. However, further studies are needed to clarify the exact role of circulating miR-25-3p in CAID.

In conclusion, for the first time, ACLF was associated with a distinct miRNA profile obtained by microarray analysis of sera from patients hospitalized for acute decompensation of cirrhosis. miR-223-3p and miR-25-3p expressions were related to creatinine levels, hepatic encephalopathy, ACLF, and mortality. Circulating miR-25-3p was an independent predictor of ACLF and short-term mortality, indicating a potential as a prognostic biomarker in patients with acute decompensation of cirrhosis.

\section{Patients and Methods}

Study population. All patients included in the present study were admitted the emergency room of the University Hospital of the Federal University of Santa Catarina, Brazil, from January 2013 to November 2015 due to acute decompensation of cirrhosis. This study was conducted in two phases. In the first phase, samples of 35 patients were used for miRNAs microarray analysis. In the second phase, miRNAs selected for validation by RT-qPCR in the first phase were investigated in samples of 139 patients. Patients in the following situations were excluded: (1) hospitalization for elective procedures; (2) admissions not related to complications of liver cirrhosis; (3) hepatocellular carcinoma outside Milan criteria; (4) extrahepatic malignancy; (5) severe extrahepatic disease.

The diagnosis of cirrhosis was established by histology (when available) or by the combination of imaging, biochemical and clinical findings in patients with evidence of portal hypertension.

This study was approved by the Ethics Committee on Human Research of the Federal University of Santa Catarina (approval number 948.198) and the study protocol was performed conform to the ethical guidelines of the 1975 Helsinki Declaration. We declare that the informed consent was obtained from all study participants and/or their legal guardians.

Patients. All patients admitted for acute decompensation of cirrhosis, as defined by the acute development of hepatic encephalopathy, large ascites, gastrointestinal bleeding, bacterial infection or any combination of these, were screened. Patients were evaluated within 24 hours of admission by one of the researchers involved in the study and followed during their hospital stay. Thirty-day mortality was evaluated by phone call, in case of hospital discharge.

Active alcoholism was defined as an average overall consumption of 14 or more drinks per week for women, and 21 or more drinks per week for men during the 4 weeks before enrollment (one standard drink is equal to $12 \mathrm{~g}$ absolute alcohol) $)^{38}$.

Hepatic encephalopathy was graded according to the West-Haven criteria ${ }^{39}$. The ACLF definition was based on the EASL-CLIF Consortium definition ${ }^{4}$, therefore, no ACLF are patients with no organ failure or with a single "non-kidney" organ failure who had a serum creatinine level less than $1.5 \mathrm{mg} / \mathrm{dL}$ and no hepatic encephalopathy, or patients with single cerebral failure who had a serum creatinine level less than $1.5 \mathrm{mg} / \mathrm{dL}$. ACLF are patients with single kidney failure, or patients with single failure of the coagulation, liver, circulation, respiration who had a serum creatinine level between 1.5 to $1.9 \mathrm{mg} / \mathrm{dL}$ and/or mild to moderate hepatic encephalopathy, or patients with single cerebral failure who had a serum creatinine level between 1.5 and $1.9 \mathrm{mg} / \mathrm{dL}$ or patients with two or more organ failures. Organ failure was established based on the CLIF-SOFA criteria ${ }^{4}$, and the severity of liver disease was estimated by the Child-Pugh ${ }^{40}$ and Model for End-Stage Liver Disease (MELD) ${ }^{41}$ classification system, calculated based on clinical and biochemical parameters obtained at admission.

Blood collection and serum isolation. Peripheral blood was collected in clot activator and separator gel (BD Vacutainer SST II Advance) tube. The blood was centrifuged at $3000 \times \mathrm{g}$ for $10 \mathrm{~min}$ and the serum was transferred to microtubes and stored at $-80^{\circ} \mathrm{C}$. Prior to use, the serum was thawed and centrifuged at $16.000 \times \mathrm{g}$ for $10 \mathrm{~min}$ at $4^{\circ} \mathrm{C}^{42}$. This step allowed the removal of cells debris and cryoprecipitate. Visibly hemolyzed, icteric or lipemic samples were not used in this study. 
RNA extraction. Total RNA was extracted using the mirVana PARIS RNA kit, according to the manufacturer instructions (Life Technologies, California, USA) using $500 \mu \mathrm{L}$ of serum. After the denaturation, $25 \mathrm{fmol}$ of synthetic miRNA cel-miR-238 ${ }^{43}$ (where "cel" corresponds to the abbreviation of species Caenorhabditis elegans) was added, which was used as quality control of RNA extraction and RT-qPCR.

miRNA microarray. miRNA expression profiling was performed using Human Agilent's miRNA Microarray system (Agilent Technologies, California, USA) with probe sets for human miRNAs (miRBase release 21). In brief, $35 \mu \mathrm{L}$ of total RNA was concentrated in SpeedVac ${ }^{\mathrm{TM}}$ Concentrator Savant SPD1010 (Thermo Fisher Scientific, Massachusetts, USA) at room temperature and vacuum pressure of approximately 10 Torr. The total RNA was fluorescence-labeled with Cyanine 3 and hybridized onto the arrays for $20 \mathrm{~h}$ at $55^{\circ} \mathrm{C}$. Slides were scanned in a SureScan Microarray Scanner (Agilent Technologies, California, USA) and the images obtained were processed with Feature Extraction Software (Agilent Technologies, California, USA). Intensity values were processed using Genespring software (Agilent Technologies, California, USA) for evaluation of the altered miRNAs. The microarray data were shown as fold change (FC) value, which describes the ratio of miRNA expression between the groups studied.

Nomenclature of miRNAs. The denomination of the miRNAs was done according to the miRBase database (www.mirbase.org) with the exception of the term "hsa" (abbreviation of species Homo sapiens) in front of the names, to avoid repetitions, as all samples are from human source.

Reverse transcription-quantitative polymerase chain reaction (RT-qPCR). Expression of selected mature miRNAs was assessed by RT-qPCR. First, $35 \mu \mathrm{L}$ of total RNA was concentrated in SpeedVac ${ }^{\mathrm{TM}}$ Concentrator Savant SPD1010 (Thermo Fisher Scientific, Massachusetts, USA) for $1 \mathrm{~h}$ at room temperature and vacuum pressure of approximately 10 Torr. The polyadenylation and cDNA were prepared from total RNA using miRNA RT-qPCR Master Mix Detection Kit (Agilent Technologies, California, USA) in a Veriti Thermal Cycler (Applied Biosystems, California, USA). The qPCR was done using a StepOnePlus Real-Time PCR System (Applied Biosystems, California, USA) to quantify the levels of cel-miR-238-3p; miR-223-3p; miR-20a-5p; miR106b-5p; miR-25-3p; miR-126-3p; and miR-1273g-3p. All reactions were run in duplicate and normalized to the expression of miR-1273g-3p. The relative expression of the miRNAs was calculated by the comparative quantification cycle $(\mathrm{Cq})$ method $^{44}$, where the $\mathrm{Cq}$ is the PCR cycle number at which the sample fluorescence signal passes a fixed threshold line, and reported as $2^{-\Delta \mathrm{Cq} \text { (miR-target - miR-1273g-3p) }}$.

Bioinformatic analysis. The bioinformatic analysis was performed using the starBase software version $2.0^{45}$. This program compiles the miRNA-mRNA interaction data from five prediction programs: TargetScan (www.targetscan.org), PicTar (pictar.mdc-berlin.de), PITA (https://genie.weizmann.ac.il/pubs/mir07/mir07_prediction. html), miRanda (www.microrna.org) and RNA22 (https://cm.jefferson.edu/rna22/Precomputed/). Furthermore, the enrichment analysis of the biological pathways was performed using the FunRich software version 3.1.3 ${ }^{46}$.

Statistical analysis. Data from miRNA microarray were analyzed by Genespring GX (Agilent Technologies, California, USA) and GraphPad Prism 6 (GraphPad Software, California, USA) software. Normality of the variable distribution was determined using the Kolmogorov-Smirnov test. The miRNAs with Fold Change (FC) $\geq 6$ were identified by the non-parametric Mann-Whitney U test with Benjamini-Hochberg correction to compare no ACLF with ACLF patients, and Storey with bootstrapping correction was used to compare patients according to the occurrence or not of death in 30 days.

Data produced from the RT-qPCR were analyzed in the IBM SPSS Statistics version 22 software (IBM, Chicago, IL, USA). The Normality of the variable distribution was also evaluated by the Kolmogorov-Smirnov test. Continuous variables were compared using Student's t-test in the case of a normal distribution or Mann-Whitney test in the remaining cases. The correlation between the numerical variables was evaluated using Spearman's correlation coefficient ( $\rho$ Spearman). Categorical variables were assessed using the chi-square test. Multiple logistic regression analysis (forward conditional method) was used to investigate factors independently associated with ACLF. Univariate and multivariate Cox regression analyses were used to investigate the association of miRNA expression with 30-day mortality. The best cutoff points for the miRNAs to predict mortality were chosen by the Receiver Operating Characteristics (ROC) curve. The Kaplan-Meier curve was used to demonstrate 30-day survival according to two strata, defined by the cutoff of selected miRNAs. Survival differences between groups were compared using the log-rank test. P-values less than 0.05 were considered statistically significant.

\section{Data availability}

The datasets generated and analyzed during the current study are contained within the manuscript and in supplementary material. Additional raw data can be available upon request.

Received: 13 August 2019; Accepted: 2 December 2019;

Published online: 09 January 2020

\section{References}

1. D’Amico, G. et al. Clinical states of cirrhosis and competing risks. J. Hepatol. 68, 563-576 (2018).

2. Jalan, R. et al. Review Acute-on chronic liver failure. J. Hepatol. 57, 1336-1348 (2012).

3. Hernaez, R., Solà, E., Moreau, R. \& Ginès, P. Acute-on-chronic liver failure: an update. Gut 66, 541 LP-553 (2017).

4. Moreau, R. et al. Acute-on-chronic liver failure is a distinct syndrome that develops in patients with acute decompensation of cirrhosis. Gastroenterology 144(1426-37), 1437.e1-9 (2013). 
5. Hernaez, R. et al. Prevalence and short-term mortality of acute-on-chronic liver failure: A national cohort study from the USA. J. Hepatol. 70, 639-647 (2019).

6. Jimenez-Lucena, R. et al. Circulating miRNAs as Predictive Biomarkers of Type 2 Diabetes Mellitus Development in Coronary Heart Disease Patients from the CORDIOPREV Study. Mol. Ther. Nucleic Acids 12, 146-157 (2018).

7. Marques, T. M. et al. MicroRNAs in Cerebrospinal Fluid as Potential Biomarkers for Parkinson's Disease and Multiple System Atrophy. Mol. Neurobiol. 54, 7736-7745 (2017).

8. Aiso, T. et al. Serum levels of candidate microRNA diagnostic markers differ among the stages of non-small-cell lung cancer. Oncol. Lett. 16, 6643-6651 (2018).

9. Amaral, A. Edo et al. MicroRNA profiles in serum samples from patients with stable cirrhosis and miRNA-21 as a predictor of transplant-free survival. Pharmacol. Res. 134, 179-192 (2018).

10. Bartel, D. P. MicroRNAs: genomics, biogenesis, mechanism, and function. Cell 116, 281-297 (2004).

11. Lee, R. C. \& Ambros, V. An extensive class of small RNAs in Caenorhabditis elegans. Science 294, 862-864 (2001).

12. Weber, J. A. et al. The microRNA spectrum in 12 body fluids. Clin. Chem. 56, 1733-1741 (2010).

13. do Amaral, A. E., Cisilotto, J., Creczynski-Pasa, T. B. \& de Lucca Schiavon, L. Circulating miRNAs in nontumoral liver diseases. Pharmacol. Res. 128, 274-287 (2018).

14. Chen, X. et al. Characterization of microRNAs in serum: a novel class of biomarkers for diagnosis of cancer and other diseases. Cell Res. 18, 997-1006 (2008).

15. Pu, C. et al. Combination of MicroRNAs and Cytokines: a Method for Better Evaluation of Acute-on-Chronic Liver Failure. Clin. Lab. 64, 247-256 (2018).

16. Wen, Y. et al. Serum levels of miRNA in patients with hepatitis B virus-associated acute-on-chronic liver failure. Hepatobiliary Pancreat. Dis. Int 17, 126-132 (2018).

17. Schueller, F. et al. miR-223 represents a biomarker in acute and chronic liver injury. Clin. Sci. (Lond). 131, 1971-1987 (2017).

18. Jin, B.-X. et al. MicroRNA panels as disease biomarkers distinguishing hepatitis B virus infection caused hepatitis and liver cirrhosis. Sci. Rep. 5, 15026 (2015).

19. Reddycherla, A. V. et al. miR-20a inhibits TCR-mediated signaling and cytokine production in human naive CD4+ T cells. PLoS One 10, e0125311 (2015).

20. Poon, K.-S. et al. Plasma exosomal miR-223 expression regulates inflammatory responses during cardiac surgery with cardiopulmonary bypass. Sci. Rep. 7, 10807 (2017).

21. Wang, H. et al. Serum microRNA signatures identified by Solexa sequencing predict sepsis patients' mortality: a prospective observational study. PLoS One 7, e38885 (2012).

22. Yao, L. et al. Clinical evaluation of circulating microRNA-25 level change in sepsis and its potential relationship with oxidative stress. Int. J. Clin. Exp. Pathol. 8, 7675-7684 (2015).

23. Chen, C. et al. Serum miR-126-3p level is down-regulated in sepsis patients. 11, 2605-2612 (2018).

24. Lee, M. S. et al. Investigated the safety of intra-renal arterial transfusion of autologous CD34+ cells and time courses of creatinine levels, endothelial dysfunction biomarkers and micro-RNAs in chronic kidney disease patients-phase I clinical trial. Oncotarget 8, 17750-17762 (2017).

25. Bhattacharya, S. et al. Serum miR-30e and miR-223 as Novel Noninvasive Biomarkers for Hepatocellular Carcinoma. Am. J. Pathol. 186, 242-247 (2016).

26. Oksuz, Z. et al. Serum microRNAs; miR-30c-5p, miR-223-3p, miR-302c-3p and miR-17-5p could be used as novel non-invasive biomarkers for HCV-positive cirrhosis and hepatocellular carcinoma. Mol. Biol. Rep. 42, 713-720 (2015).

27. Wang, T.-Z., Lin, D.-D., Jin, B.-X., Sun, X.-Y. \& Li, N. Plasma microRNA: A novel non-invasive biomarker for HBV-associated liver fibrosis staging. Exp. Ther. Med. 17, 1919-1929 (2019).

28. Li, L.-M. et al. Serum microRNA Profiles Serve as Novel Biomarkers for HBV Infection and Diagnosis of HBV-Positive Hepatocarcinoma. Cancer Res. 70, 9798 LP-9807 (2010).

29. Wasmuth, H. E. et al. Patients with acute on chronic liver failure display 'sepsis-like' immune paralysis. J. Hepatol. 42, 195-201 (2005).

30. Yao, Y., Sun, F. \& Lei, M. miR-25 inhibits sepsis-induced cardiomyocyte apoptosis by targetting PTEN. Biosci. Rep. 38 (2018)

31. Ye, D., Zhang, T., Lou, G. \& Liu, Y. Role of miR-223 in the pathophysiology of liver diseases. Exp. Mol. Med. 50, 128 (2018).

32. Li, M. et al. MicroRNA-223 ameliorates alcoholic liver injury by inhibiting the IL-6-p47 $7^{\text {phox }}$-oxidative stress pathway in neutrophils. Gut 66, 705 LP-715 (2017).

33. He, Y. et al. MicroRNA-223 Ameliorates Nonalcoholic Steatohepatitis and Cancer by Targeting Multiple Inflammatory and Oncogenic Genes in Hepatocytes. Hepatology 70, 1150-1167 (2019).

34. Ulbing, M. et al. MicroRNAs 223-3p and 93-5p in patients with chronic kidney disease before and after renal transplantation. Bone 95, 115-123 (2017).

35. Bruno, N. et al. MicroRNAs relate to early worsening of renal function in patients with acute heart failure. Int. J. Cardiol. 203, 564-569 (2016).

36. Gholaminejad, A., Abdul Tehrani, H. \& Gholami Fesharaki, M. Identification of candidate microRNA biomarkers in diabetic nephropathy: a meta-analysis of profiling studies. J. Nephrol. 31, 813-831 (2018).

37. Albillos, A., Lario, M. \& Alvarez-Mon, M. Cirrhosis-associated immune dysfunction: distinctive features and clinical relevance. J. Hepatol. 61, 1385-1396 (2014).

38. Sanyal, A. J. et al. Endpoints and clinical trial design for nonalcoholic steatohepatitis. Hepatology 54, 344-353 (2011).

39. Bajaj, J. S. Review article: the modern management of hepatic encephalopathy. Aliment. Pharmacol. Ther. 31, 537-547 (2010).

40. Angermayr, B. et al. Child-Pugh versus MELD score in predicting survival in patients undergoing transjugular intrahepatic portosystemic shunt. Gut 52, 879-885 (2003).

41. Kamath, P. S. et al. A model to predict survival in patients with end-stage liver disease. Hepatology 33, 464-470 (2001).

42. Zhou, J. et al. Plasma microRNA panel to diagnose hepatitis B virus-related hepatocellular carcinoma. J. Clin. Oncol. 29, 4781-4788 (2011).

43. Cermelli, S., Ruggieri, A., Marrero, J. A., Ioannou, G. N. \& Beretta, L. Circulating microRNAs in patients with chronic hepatitis C and non-alcoholic fatty liver disease. PLoS One 6, e23937 (2011).

44. Livak, K. J. \& Schmittgen, T. D. Analysis of relative gene expression data using real-time quantitative PCR and the 2(-Delta Delta C(T)) Method. Methods 25, 402-408 (2001).

45. Li, J.-H., Liu, S., Zhou, H., Qu, L.-H. \& Yang, J.-H. starBase v2.0: decoding miRNA-ceRNA, miRNA-ncRNA and protein-RNA interaction networks from large-scale CLIP-Seq data. Nucleic Acids Res. 42, D92-7 (2014).

46. Pathan, M. et al. FunRich: An open access standalone functional enrichment and interaction network analysis tool. Proteomics 15, $2597-2601(2015)$.

\section{Acknowledgements}

This work was supported by grants from Conselho Nacional de Desenvolvimento Científico e Tecnológico (CNPq, Grant No. 457373/2013-0), Coordenação de Aperfeiçoamento de Pessoal de Nível Superior (CAPES), and Fundação de Amparo à Pesquisa e Inovação do Estado de Santa Catarina (FAPESC). 


\section{Author contributions}

J.C., A.E.A., D.R., A.H.S., E.W., L.L.S. and T.B.C.-P. contributed to the design of the study. J.C., A.E.A., D.R. and M.P.R. contributed to the experiments and data analysis. J.C., T.B.C.-P. and L.L.S. contributed to data interpretation and data analysis. T.E.S., J.F., C.M., E.C.M.R. and J.L.N.S. collected and organized the samples and performed the clinical tests. J.C., L.L.S. and T.B.C.-P. wrote the manuscript. All authors reviewed the manuscript and contributed to scientific discussions.

\section{Competing interests}

The authors declare no competing interests.

\section{Additional information}

Supplementary information is available for this paper at https://doi.org/10.1038/s41598-019-56630-5.

Correspondence and requests for materials should be addressed to L.d.L.S. or T.B.C.-P.

Reprints and permissions information is available at www.nature.com/reprints.

Publisher's note Springer Nature remains neutral with regard to jurisdictional claims in published maps and institutional affiliations.

(c) (i) Open Access This article is licensed under a Creative Commons Attribution 4.0 International License, which permits use, sharing, adaptation, distribution and reproduction in any medium or format, as long as you give appropriate credit to the original author(s) and the source, provide a link to the Creative Commons license, and indicate if changes were made. The images or other third party material in this article are included in the article's Creative Commons license, unless indicated otherwise in a credit line to the material. If material is not included in the article's Creative Commons license and your intended use is not permitted by statutory regulation or exceeds the permitted use, you will need to obtain permission directly from the copyright holder. To view a copy of this license, visit http://creativecommons.org/licenses/by/4.0/.

(c) The Author(s) 2020 